\title{
PADRONIZAÇÃO DA INFORMAÇÃO GEOGRÁFICA E METADADOS GEOESPACIAIS DIGITAIS
}

\author{
GILBERTO PESSANHA RIBEIRO* \\ Universidade Federal Fluminense e \\ Universidade do Estado do Rio de Janeiro
}

A informação geográfica, relativa aos fenômenos geográficos que possuem referência espacial, consiste em informação quantitativa e qualitativa relativa a objetos e fenômenos de natureza física e humana, distribuída espacialmente pela superfície terrestre. A manipulação e o tratamento da informação geográfica em um contexto de Bancos de Dados e SIG não é tarefa trivial, principalmente diante do volume e da complexidade dos dados geográficos (MARTIN et al., 1991). Pesquisas recentes na área de Informática, especialmente na linha de Banco de Dados, apontam algumas soluções para a manipulação desses dados. Dentre essas pesquisas destacam-se: banco de dados temporais, interfaces adaptativas de banco de dados, mineração de dados, trabalho cooperativo e fluxo de tarefas em um ambiente de bancos de dados múltiplos, dificuldades na interoperabilidade entre sistemas de gerência de banco de dados, aspectos de heterogeneidade (conflitos) entre bases de dados distribuídas e impacto do estabelecimento de padrões de metadados nas arquiteturas internas de bancos de dados.

As questões levantadas e que motivam o desenvolvimento desse trabalho são as seguintes: quais são os requisitos necessários para recuperação de dados por um gerenciador de metadados? Em que arquitetura deve esse gerenciador operar? De que forma deve ser medida a performance do sistema? Como integrar metadados geográficos em um ambiente distribuído e heterogêneo? O sistema a ser descrito nesse trabalho pode ser desenvolvido, em atendimento às necessidades das aplicações SIG e à complexidade dos dados geográficos. O projeto, o estudo e a análise desse sistema apresentam contribuições nas seguintes direções:

- Introdução de mecanismos para indexação e consulta a dados externos, para o projeto

\footnotetext{
* Engenheiro Cartógrafo (UERJ), Mestre em Ciências Geodésicas (UFPR), Doutorando junto ao Programa de Pós-Graduação em Geografia da UFF, Professor Adjunto do Departamento de Cartografia da Universidade Federal Fluminense (UFF) e Professor Assistente do Departamento de Engenharia Cartográfica da Faculdade de Engenharia da Universidade do Estado do Rio de Janeiro (UERJ).E-mail: gilberto@vm.uff.br e pessanha@uerj.br. O autor registra um especial agradecimento a Ivan de Araújo Medina e a Mauro Pereira de Mello, professores da UERJ, pelo incentivo freqüente e pela relevante troca de idéias e experiências durante as pesquisas realizadas. Também agradece à engenheira cartógrafa Anna Lucia Barreto de Freitas, Gerente do Projeto de Informatização da Cartografia (INFOCAR) do Departamento de Cartografia da Fundação IBGE e à geógrafa Dra. Marli Cigagna, do Departamento de Cartografia da UFF, pelas expressivas sugestões e estímulos freqüentes na busca de análise e pesquisas que culminam em discussões valiosas sobre temas contemporâneos aplicáveis à área de Cartografia e de Geografia, respectivamente.
} 
físico e sobre os dados dos repositórios externos;

- Introdução de um mecanismo simples, "semelhante" para tipos desconhecidos, que forneça um tratamento mais uniforme aos tipos de dados abstratos em bancos de dados, através de um número restrito de conceitos; e

- Discussão do impacto sobre as arquiteturas dos bancos de dados internos, dando uma demonstração prática do caso estudado através do próprio exemplo de extensão do sistema.

De fato, a administração de metadados coloca exigências especiais aos gerenciadores, o que faz os bancos de dados tradicionais serem incapazes de resolvê-los facilmente. Os seguintes pontos, no desenvolvimento desse trabalho, estão sendo alvo de estudos:

- Qualquer categoria de informação (gráfica ou não) deve ser descrita nos padrões propostos e previamente estabelecidos;

- $\quad$ Qualquer consulta deve ser apoiada por metadados;

- Dados "semelhantes" devem ser suportados pelo modelo de dados; e

- Necessidade de criar instrumentos necessários para que sirvam de apoio na "junção" de dados ditos "semelhantes".

Um aspecto muito importante para o desenvolvimento de bases de dados de utilização por diversos grupos de usuários, com múltiplos interesses, é a documentação de seu conteúdo. Sem documentação apropriada toma-se difícil para os usuários localizar os dados necessários para as suas aplicações, bem como entender seu significado. Uma vez encontrados os dados, normalmente é necessário conhecer como foram coletados e que acurácias possuem. As descrições desses dados armazenados são comumente denominadas de metadados.

Um dicionário de dados pode ser usado para organizar os metadados. Ele poderá conter uma seção descrevendo, numa visão geral, como os dados são subdivididos em arquivos, que campos de registros se relacionam, e possuir tópicos tais como convenções adotadas em sua definição. Uma seção principal desse dicionário de dados deveria conter os metadados assim como as descrições de cada campo. Para cada campo, os seguintes ítens poderiam ser incluídos (JACOBSON, 1994): nome do campo; descrição do campo; tipo de dados; formato; métodos da coleção de dados; acurácia; unidades; ou qualquer outra informação que julgue se relevante.

Organizações nos Estados Unidos, no Canadá e na Europa, por exemplo, já estabeleceram padrões uniformes de MGD. Esses padrões cobrem conteúdo e semântica dos metadados, incluindo sua documentação detalhada e também sua representação digital. Esses metadados são atualmente utilizados com níveis variados de detalhes, abordando, numa visão geral, os seguintes tópicos:

- $\quad$ Controle de qualidade: metadados detalhados são essenciais para permitir a garantia de qualidade da informação nas organizações que produzem e mantém grandes bases de dados. Os metadados documentam por completo o ciclo-de-vida dos dados, incluindo sua coleção, pré-processamento e manutenção. Esse tipo de metadados normalmente é muito pormenorizado;

- Intercâmbio de dados: devido ao alto custo na coleta e aquisição de dados geográficos, por meio de levantamentos específicos, o intercâmbio desses dados é encorajado pelas organizações e promovido pelos produtores desses dados, tornando-se uma regra geral no processamento da informação geoespacial. Os dados geográficos que faltam ou que são mal interpretados, representam uma das fontes de erros e de incertezas quando se trata do 
uso de Bancos de Dados externos às organizações. Os detalhes encontrados nos metadados, relativos a grandes bases de dados, contribuem para evitar esses tipos de problema; e

- Diretórios de dados: a necessidade de reuso (reutilização) de conjuntos de dados caros motiva o estabelecimento de diretório de dados. Eles permitem que os usuários conheçam e acessem as descrições de conteúdo de conjuntos de dados, sem ser necessário acesso aos dados propriamente ditos a partir de sua base. Um exemplo típico: usuários estão interessados em conhecer e localizar dados para uma determinada região geográfica. Diretórios devem ser criados pelas organizações contendo descrições do conteúdo dos metadados de bibliotecas digitais a partir de suas fontes. Esses metadados tipicamente requerem um nível baixo de minúcias.

Os metadados se ocupam com os requisitos relativos ao armazenamento e à consulta, independentemente dos dados primários contidos nas suas bases. Os SIG normalmente oferecem meios de armazenamento de algumas descrições das bases de dados. Essas descrições são referentes à lista de temas e suas regiões ou localizações geográficas. Estudos têm sido feitos no sentido de encontrar um melhor entendimento e clareza dos requisitos básicos de um gerenciador de metadados geográficos, de um modelo apropriado para representação desses dados e de um sistema de armazenamento de dados eficiente. Quais são os requisitos para recuperação desses dados por um gerenciador? Em que contexto de arquitetura deve esse gerenciador operar? Como pode um SGBD ser estendido para atender a esses requisitos? Blott et al. (1996) propõe respostas a essas perguntas.

O volume e a complexidade dos dados geográficos em geral, relativos às diversas aplicações SIG, contribuem para a tendência mundial direcionada ao estudo e ao estabelecimento de padrões da informação geográfica e de conteúdos de metadados para esse propósito. Esses dados, normalmente, provêm de levantamentos de dados de relevo (Sensoriamento Remoto, Topografia Clássica, Geodésia Clássica, Fotogrametria, Sistema de Posicionamento Global, etc...), de população (censos econômicos, agropecuários, demográficos, etc...) e de ambiente (coleta de dados físicos meteorológicos, etc...).

French (1992) apresenta duas classes gerais de metadados, que descrevem:

- $\quad$ Características físicas ou estruturais dos dados: permite decodificar bits em valores inteiros, reais e outras estruturas. Por exemplo, conversão de um arquivo no formato binário (linguagem de máquina) para o formato ASCII, sem perda de informação, destinado à impressão;

- Interpretação lógica dos dados: necessidade de localizar dados, em um determinado contexto, e de permissão para a sua interpretação.

Podem ser propostos: conteúdo, qualidade, formatos e condições de uso de dados geográficos, necessários para o desenvolvimento de projetos multidisciplinares para o uso em Sistemas de Informação Geográfica (SIG). Esses padrões especificam o conteúdo das informações da base de metadados para um conjunto de dados geográficos digitais. Padrões já foram e estão sendo propostos em países de economia avançada, como os Estados Unidos e o Canadá, e benefícios concretos têm surgido com a sua adoção. Os padrões a serem, de forma introdutória, comentados aqui e alguns de seus elementos especificados se referem à informação de conteúdo para um conjunto de dados geográficos digitais. O propósito para o estabelecimento desses padrões é fornecer terminologia e definições comuns para conceitos relacionados a esses 
metadados geográficos.

Um ponto de partida para coordenar a aquisição e o acesso a dados geográficos pode estar na determinação de uma infraestrutura de dados espaciais, a princípio em âmbito nacional, mas com possibilidade de globalização, num esforço conjunto pelas instituições depositárias desses dados. Várias aplicações e organizações podem ser beneficiadas por esses padrões. A ordem na qual os elementos dos dados são avaliados pelas empresas e a relativa importância dos elementos dos dados não serão as mesmas para todos os usuários, ou para todos os projetos que usam os metadados. Usuários de diferentes projetos, ou em diferentes estágios de avaliação, podem requerer ou preferir que um conjunto de informações esteja disponível, a diferentes níveis de abstração, ou em diferentes formas.

A função desses padrões de MGD será, basicamente, fornecer as definições e formar uma rede para automatizar registros de propriedades e dados cadastrais, em uma forma padrão, em todos os níveis governamentais e também no setor privado. Os padrões resultantes poderão acomodar a automação dos dados exatamente como eles se encontram no registro público.

Os objetivos são obter resultados onde se procura:

- $\quad$ Fornecer definições comuns para a informação cadastral encontrada em registros públicos para facilitar o seu uso efetivo, o seu entendimento e, obviamente, a automação desses registros de propriedades;

- Valores de atributos padronizados permitindo um bom compartilhamento de dados;

- Resolver discrepâncias relacionadas ao uso de homônimos e sinônimos em sistemas de registro de propriedades federais para minimizar duplicação com e entre aqueles sistemas;

- $\quad$ Fornecer guia e direção através da documentação de atributos padronizados e definições para aperfeiçoar a criação, gerência e uso de registro de propriedades;

- Usar de forma participativa os padrões e se envolver, direta e indiretamente, com o desenvolvimento de padrões para que possam ser alcançadas as organizações em geral, além de sensibilizar as organizações não governamentais e encorajá-las a desenvolver aplicações baseadas nesses padrões;

- Desenvolver procedimentos padronizados e processos para coleção de dados cadastrais minimizando a duplicação de esforços individuais e coletivos; e

- Desenvolver um perfil cadastral para metadados geográficos digitais permitindo a inclusão de dados cadastrais nas agências federais de dados espaciais.

\section{Uma apresentação geral de alguns padrões de conteúdo de metadados que coexistem hoje}

O Spatial Archive and Interchange Format (SAIF) foi desenvolvido por um órgão governamental canadense (Surveys and Resource Mapping Branch, Ministry of Environment, Lands and Parks - MELP) como um meio de compartilhamento de dados espaciais e espaçotemporais. Segundo o MELP (1994), seus objetivos principais se relacionam com um padrão que deve: ser apropriado para modelar e mover dados, isto é, deve ser capaz de lidar com informações espaço-temporais e tradicionais; manipular virtualmente qualquer tipo de dado geográfico, incluindo aqueles com ou sem descrição de atributos, e com geometria definida por estruturas vetoriais ou matriciais, em duas ou em três dimensões; endereçar tempo de forma que eventos 
temporais e relacionamentos possam ser manipulados; endereçar requisitos de gerência de dados tais como: suportar atualizações, habilidade de integração com dados multimídia, aplicabilidade para manipular grandes e pequenos volumes de dados, habilidade para uma interface boa com consultas de bancos de dados e compatibilidade com desenvolvimento de catálogos; ser adaptável para operações efetivas em ambientes de redes de comunicação de dados, tão bem como apropriado para ser usado com um sistema de transferência de arquivo convencional por meio ótico ou magnético; ser de fácil uso e de baixo custo, além de possuir fácil manutenção e extensão, em resposta para as necessidades do usuário e mudanças de tecnologia; e ser capaz de possuir compatibilidade e harmonia com novos desenvolvimentos de consultas de Bancos de Dados e aplicações SIG; tão bem como outros padrões geográficos.

O SAIF está num formato orientado a objetos e foi originalmente desenvolvido para modelar, armazenar, recuperar e permitir intercâmbio de dados espaço-temporais (MURRAY et al., 1994). Dentre suas características, destacam-se: permite herança múltipla no modelo de dados; é extensível, permitindo aos usuários definir classes e herança a partir de muitas classes cásicas que são definidas em sua especificação; reconhece que a única maneira prática de processar grandes volumes de dados em tempo real é através do uso do paralelismo, isto é, computação paralela; habilita os chamados Binary Large Objects (BLOB's), como imagens, sons ou animações, a se "encaixar" isto é, a serem tratados eficazmente em um conjunto de dados; é independente da plataforma, isto é, um único conjunto de dados pode ser usado sobre múltiplas plataformas de hardware e software; e os conjuntos de dados SAIF têm uma densidade de informação muito alta resultando em uso eficiente de armazenamento e transmissão de dados a partir de suas fontes. O modelo de dados do SAIF, como já foi dito, permite o uso de herança múltipla, no paradigma de orientação a objetos, permitindo aos usuários facilmente criar classes usando técnicas de orientação a objetos, assim como acontece com linguagens de programação orientadas a objetos. Ele apresenta um modelo genérico conceitual com 300 classes de objetos geográficos envolvendo dados gráficos e não gráficos.

Já para o estabelecimento do padrão estadunidense Spatial Data Transfer Standard (SDTS), foi formado um comitê por instituições governamentais dos EUA, dentre elas: Departamentos de Estado, Comércio, Defesa, Energia, Desenvolvimento Urbano, Interior, Agricultura, Transportes, Agências de Proteção de Meio Ambiente, de Gerência de Emergência Federal, Biblioteca do Congresso, Administração Espacial e Aeronáutica Nacional (NASA) e Administração Nacional de Registros e Arquivos.

Esforços conjuntos levaram à constituição de um padrão para os metadados geográficos digitais através desse comitê. Os maiores usos desses metadados, analisados a partir dos padrões já estabelecidos encontrados em (FGDC, 1994a, e FGDC, 1994b), são, em resumo: auxiliar na organização e manutenção de investimentos internos em dados espaciais; fornecer informação sobre dados da organização através de catálogos e agências de coleta, distribuição de informação e atendimento a usuários (FGDC, 1994c); e fornecer informação para processar e interpretar dados, recebidos através de um sistema, a partir de uma fonte externa.

A informação a ser incluída nesses padrões deve ser selecionada com base em quatro características que definem parte dos metadados: disponibilidade: dados necessários para determinar os conjuntos de dados que existem para uma localização geográfica; adequabilidade: dados necessários para determinar se um conjunto de dados satisfaz a uma necessidade especificada; acesso: dados necessários para adquirir um conjunto especificado de dados; e 
transferência: dados necessários para transferência, processamento e uso de um conjunto de dados. Essas características levam ao usuário a determinar que dados estão disponíveis, a avaliar a adequabilidade dos dados para o uso, e, por último, a transferir e processar dados em geral.

O Comité Européen de Normalisation (CEN) já propõe, no contexto das aplicações geográficas, um modelo composto por seções que descrevem modelo de referência, esquema espacial, qualidade, metadados, transferência, posição espacial (georreferência), consulta e atualização, além de identificadores espaciais. Alguns pseudocódigos são apresentados e existe um modelo de referência para descrever dados geográficos onde são tratados os seguintes assuntos: sistema de referência espacial, thesaurus, tipo de objeto abstrato, dados de fontes, acurácia, qualidade e atualidade dos dados, etc... Esse padrão tem sido referência para os estudos e trabalhos voltados para o estabelecimento de padrões de conteúdo para metadados geográficos.

\section{Concert: um sistema, do tipo protótipo, desenvolvido para gerenciar metadados}

Como exemplo de um sistema de gerenciamento de metadados pode ser citado o CONCERT (BLOTT et al., 1995). Esse sistema consiste de uma extensão de banco de dados voltada para o armazenamento de metadados baseado em objetos abstratos. Os objetivos desse sistema são alcançar um entendimento melhor dos requisitos básicos para um gerenciador de metadados e testar ao máximo um modelo de armazenamento avaliando o seu desempenho.

As questões levantadas e que motivaram o desenvolvimento do protótipo CONCERT são as seguintes: quais são os requisitos necessários para recuperação de dados por um gerenciador de metadados? Em que arquitetura deve esse gerenciador operar? A solução de arquitetura encontrada por aquele autor pode ser vista na Figura 2.

Esse protótipo apresenta contribuições nas seguintes direções:

- Introdução de mecanismos para indexação e consulta a dados externos, para o projeto físico e sobre os dados dos repositórios externos;

- Introdução de um mecanismo simples, "semelhante" para tipos desconhecidos, que forneça um tratamento mais uniforme aos Abstract Data Tytes (ADT) em bancos de dados, através de um número restrito de conceitos; e

- Discussão do impacto sobre as arquiteturas dos bancos de dados internos, dando uma demonstração prática do caso estudado através do próprio exemplo de extensão do sistema.

De fato, a administração de metadados coloca exigências especiais aos gerenciadores, o que faz os bancos de dados tradicionais serem incapazes de resolvê-los facilmente.

Os seguintes pontos estão sendo alvo de estudos, no contexto do CONCERT, segundo os relatos de Etter (1995):

- Qualquer categoria de informação (gráfica ou não) deve ser descrita nos padrões;

- $\quad$ Qualquer pergunta (consulta) deve ser apoiada por metadados;

- Dados "semelhantes" devem ser suportados pelo modelo do CONCERT; e

- Instrumentos necessários para que sirvam de apoio na "junção" de dados ditos "semelhantes".

É eminente a necessidade de se conhecer melhor o desempenho de sistemas já desenvolvidos ou em desenvolvimento com objetivos de gerenciar metadados geográficos digitais. O protótipo CONCERT mostra uma solução alternativa na gerência dos metadados. 
Uma alternativa para solução desse problema pode ser testada com o apoio de um sistema gerenciador de banco de dados orientado a objetos, como por exemplo, o sistema 02 que se encontra disponível na COPPE-UFRJ, no contexto do Programa de Engenharia de Sistemas e Computação. Essa será a próxima etapa a ser vencida nesse trabalho.

Além disso, os padrões propostos pelo SDTS e SAIF devem ser avaliados criteriosamente como proposto por CRISPIM (1991), segundo dois objetivos: o objetivo "utilizabilidade" para construção (aplicabilidade, expressividade, facilidade de aprendizado para construção e facilidade de uso) e o objetivo "utilizabilidade" para avaliação (verificabilidade e valiabilidade). Essa avaliação global dos padrões deve ser feita de forma aprofundada, e torna-se decisiva para a adoção parcial ou total dos padrões de conteúdo para os MGD, para a realidade brasileira dos Bancos de Dados Federais, em um ambiente distribuído e heterogêneo. Andjelic et al. (1996) apresentam, por exemplo, um gerenciamento de versões para SIG distribuídos em um ambiente de bancos de dados múltiplos. A solução proposta por esse autor realça a necessidade da manutenção da consistência dos dados e a integração entre os SIG e os sistemas de bancos de dados múltiplos, além de integração dos ambientes das transações. A implementação de um modelo de integração é por ele apresentada em seu recente trabalho.

\section{Comentários e conclusões}

Bancos de Dados tradicionais são alvo de pesquisas há vários anos no Brasil, principalmente para aplicações comerciais. Em aplicações científicas estudos têm surgido, há algum tempo, no sentido de analisar as características dos requisitos básicos para o desenvolvimento de Bancos de Dados para suportar sistemas aplicativos de informação não convencionais (RIBEIRO, 1995a).

Bancos de Dados para as aplicações geográficas são alvo de investigações por técnicos especializados em Informática. $\mathrm{O}$ trabalho aqui apresentado não pretende, de forma alguma, esgotar uma discussão sobre os padrões comentados. Ele pretende fomentar discussões e questões importantes para o estabelecimento desses padrões de metadados, a nível nacional, apontando potenciais estudos de casos brasileiros.

Esse artigo aborda questões importantes relacionadas à organização, ao acesso e ao uso efetivo dos dados geográficos, além de proporcionar discussões que possam levar a um amadurecimento de tópicos relativos ao tema, sempre de forma cooperativa.

Instituições federais responsáveis pela manutenção de Bancos de Dados Geográficos e Cartográficos podem e devem receber a colaboração de outras instituições governamentais (estaduais e municipais) e também do setor privado. Parece ser viável a criação de um comitê nacional que se responsabilize pela manutenção dos metadados e delibere tecnicamente sobre questões levantadas sobre essa tecnologia. Maiores aprofundamentos sobre os conceitos e as definições referentes às especificações dos metadados geográficos digitais poderão ser feitos.

Uma infraestrutura de dados geográficos no Brasil pode ser estabelecida nos moldes da realidade brasileira. Essa infraestrutura pode ter como base aquela proposta por MSC (1991) que foi validada para os Estados Unidos. Alguns tópicos importantes a serem levantados e discutidos são: o estado atual das instituições brasileiras provedoras de dados geográficos; geração de dados urbanos e rurais cadastrais; compartilhamento de dados desse tipo e apontar soluções parciais para intercâmbio de dados por meios eletrônicos. Recomendações podem surgir a partir de 
reuniões técnicas de grupos categorizados de trabalho. Por exemplo, os dados cartográficos podem ser classificados nas seguintes categorias, como sugere IBGE (1995): hipsografia, limite, ponto de referência, hidrografia, vegetação, localidade, sistema viário e obra e edificação. Os grupos de trabalho poderão se envolver com temas específicos, como já estabelecido por FGDC (1995), conforme Tabela 1.

Bibliotecas cartográficas digitais têm sido desenvolvidas no sentido de solucionar problemas de gerência de dados geográficos e de acesso a bases de dados em um ambiente distribuído, por meio de redes de comunicação de dados. O Projeto Alexandria é um exemplo estadunidense que pode ser referência para outros projetos semelhantes a serem desenvolvidos no Brasil (SMITH, 1995).

Dentre os princípios de operação de um esquema para o grupo de trabalho, que comporia esse comitê nacional, deveria se considerar que:

- $\quad$ Metadados usados nesse contexto serão armazenados em meios digitais;

- $\quad$ Novos dados geográficos deverão ser documentados com base nesses padrões;

- Uma fase de adaptação para a inclusão dos dados geográficos poderá ser prevista;

- $\quad$ Produtores de dados geográficos deverão ser responsáveis pela criação dos metadados referentes aos dados que produzem;

- Mecanismos para a disseminação de dados geográficos poderão ser criados por meio de bureaus ou agências;

- $\quad$ O esquema poderá fazer uso de padrões de processamento de informação federal, além de suportar o desenvolvimento de novos padrões de dados que herdem o compartilhamento entre dados geoespacias e a habilidade para formar sociedades de dados geográficos;

- O esquema poderá assegurar acesso aos metadados através de meios eletrônicos, incluindo o uso de redes de comunicação de dados.

Regras e responsabilidades para todos os componentes do grupo de trabalho poderão ser estabelecidas e procedimentos para a documentação e compartilhamento dos dados geográficos poderão ser testados em várias situações pelas instituições. Relatos de experiências no Reino Unido, nesse sentido, podem ser encontradas no trabalho de Rhind (1996). Cada bureau poderá determinar individualmente como os produtores de dados geográficos, gerentes de dados, gerentes de SIG, administradores de bibliotecas digitais, especialistas em gerência de fonte de informação, pessoal de assuntos públicos e outros, assegurarão que os objetivos do esquema do grupo de trabalho sejam claramente atingidos.

Acredita o autor que universidades possam contribuir positivamente, enquanto academias geradoras e usuárias de tecnologias e órgãos de pesquisa científica, para os estudos voltados para o estabelecimento de modelos de dados a serem adotados e para o estabelecimento dos padrões desses metadados.

Os modelos do SAIE, do FGDC e do CEN mostram que há conceitos e padrões bem estruturados que podem ser adaptados para as aplicações SIG no Brasil, onde benefícios podem ser extraídos a partir das experiências bem sucedidas daqueles países estrangeiros.

Aspectos como heterogeneidade e inter-operabilidade de Sistemas de Gerência de Bancos de Dados (SGBD) para aplicações geográficas devem ser considerados e analisados no estabelecimento desses padrões (STRAUCH, 1996).

O autor ressalta a necessidade de discussão aprofundada sobre conceitos existentes nos 
padrões estrangeiros já estabelecidos, do ponto de vista do rigor cartográfico. No caso brasileiro é imperativa a geração de um conjunto mínimo de elementos cartográficos digitais que dê suporte às aplicações geográficas a serem desenvolvidas a partir dos padrões.

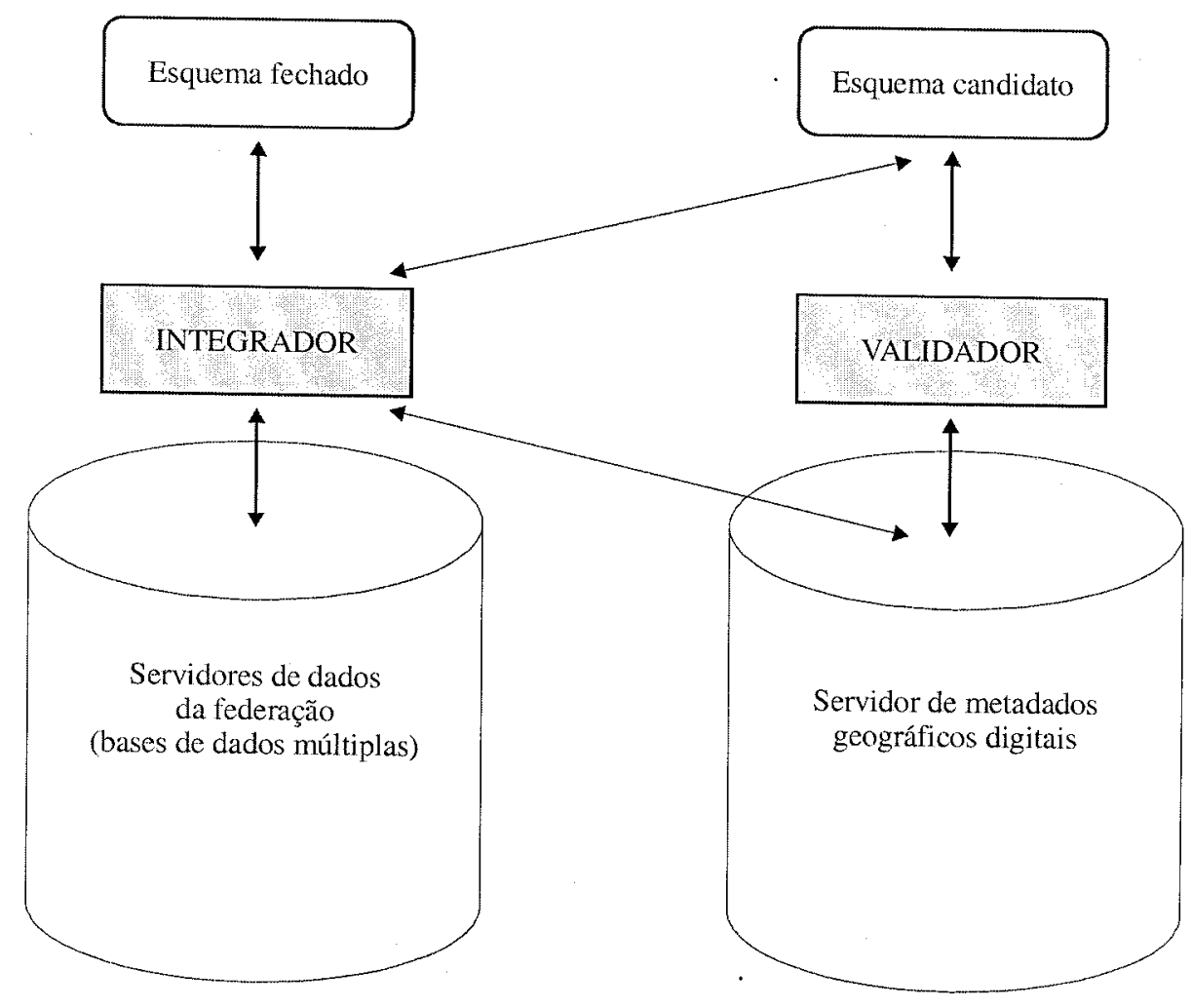

Principais serviços:

- Consulta e atualização de dados

- Transferência de dados

Figura 1: Componentes responsáveis pela manipulação de metadados geográficos digitais na federação (ambiente de bancos de dados múltiplos). 


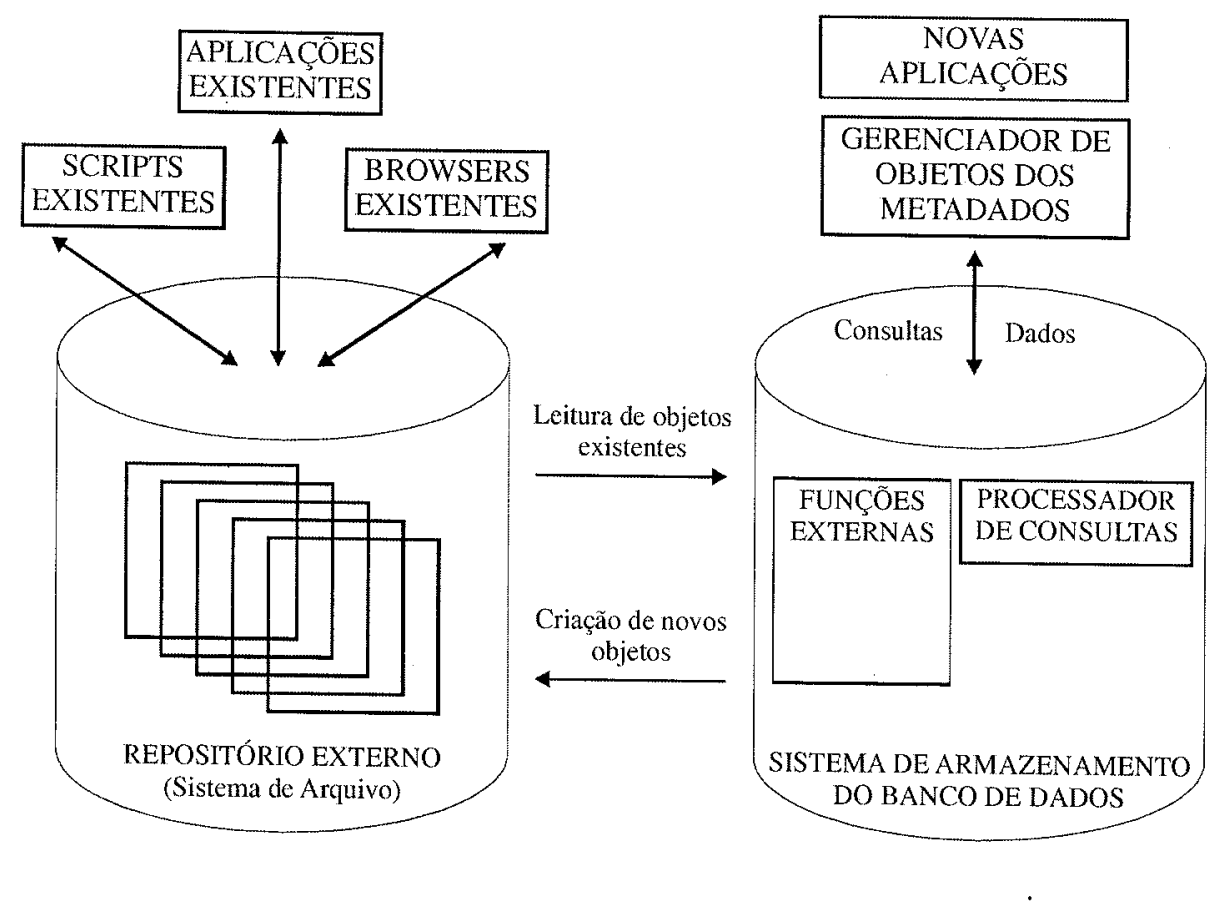

Figura 2: Solução de arquitetura para o sistema aberto para armazenamento de objetos abstratos e acesso a metadados geográficos digitais.

Tabela 1: No contexto do Comitê Geral FDGC existem sub-comitês destinados ao tratamento da informação geográfica, em âmbito nacional, nos Estados Unidos.

\begin{tabular}{|l|l|}
\hline \multicolumn{1}{|c|}{ GRUPOS } & \multicolumn{1}{|c|}{ TEMA CENTRAL } \\
\hline $\begin{array}{l}\text { Base } \\
\text { Cartográfica }\end{array}$ & $\begin{array}{l}\text { Responsável pela coordenação de atividades relacionadas com um } \\
\text { determinado tema ou categoria especifica de dado geoespacial, incluindo } \\
\text { padrões para ortoimagens digitais e dados de elevações (altitudes) digitais. } \\
\text { Atividades estas em conformidade com o Plano Cartográfico Nacional. }\end{array}$ \\
\hline Batirmetria & $\begin{array}{l}\text { Em cooperação com a Organização Hidrográfica Internacional (IHO), esse } \\
\text { grupo pode ser responsável pelos padrões de acurácia e de qualidade de } \\
\text { dados batimétricos. }\end{array}$ \\
\hline Cadastro & $\begin{array}{l}\text { Descrição e documentação de elementos contidos no tema cadastro, suas } \\
\text { definições, seus atributos e seus relacionamentos. }\end{array}$ \\
\hline $\begin{array}{l}\text { Cultura e } \\
\text { Demografia }\end{array}$ & $\begin{array}{l}\text { Dados demográficos e culturais que se encontrem com as necessidades de } \\
\text { gerência e planejamento nacionais e com a identificação de esquemas de } \\
\text { classificação para esses dados. }\end{array}$ \\
\hline
\end{tabular}




\begin{tabular}{|l|l|}
$\begin{array}{l}\text { Controle } \\
\text { Geodésico } \\
\text { Federal }\end{array}$ & $\begin{array}{l}\text { Responsável pelos padrões de dados geodésicos, incluindo padrões } \\
\text { estabelecidos pelo Sistema Geodésico Brasileiro. }\end{array}$ \\
\hline $\begin{array}{l}\text { Transportes } \\
\text { Terrestres }\end{array}$ & $\begin{array}{l}\text { Responsável pelo desenvolvimento de padrões para facilitar o intercâmbio } \\
\text { entre bases de dados geográficos relativos a redes de transporte. }\end{array}$ \\
\hline $\begin{array}{l}\text { Limites de } \\
\text { Fronteiras } \\
\begin{array}{l}\text { Internacionais } \\
\text { \& Soberania } \\
\text { Nacional }\end{array}\end{array}$ & $\begin{array}{l}\text { Responsável pelo desenvolvimento de bases de dados de vizinhança } \\
\text { (fronteira) em concordância com o Estado Maior das Forças Armadas } \\
\text { (EMFA) os Ministérios do Exército, Marinha e Aeronáutica brasileiros. }\end{array}$ \\
\hline
\end{tabular}

PADRONIZAÇÃO DA INFORMAÇÃO GEOGRÁFICA E METADADOS GEOESPACIAIS DIGITAIS

Resumo: Um sistema gerenciador de banco de dados pode possuir uma extensão de um banco de dados voltada para o armazenamento e recuperação de metadados baseados em objetos abstratos. O objetivo deste trabalho é alcançar um entendimento melhor dos requisitos básicos para um gerenciador de metadados. operar satisfatoriamente, além de testar ao máximo um modelo de armazenamento avaliando o seu desempenho, diante dos diversos tipos de dados geográficos existentes. O trabalho aponta a necessidade de se conhecer melhor o desempenho de sistemas já desenvolvidos, ou em desenvolvimento, com objetivos de gerenciar metadados geográficos digitais.

Palavras-chave: Sistemas de Informação Geográfica, Geoprocessamento, Geografia, Bancos de Dados, Computação.

\section{STANDARDIZATION OF GEOGRAPHIC INFORMATION AND DIGITAL GEOSPATIAL METADATA}

Summary: Database management systems can be design with an extended database in order to store and retrieve metadata based on abstract objects. The target of this paper is a better understanding of basic requirements to this database management system operates satisfactorily, above all to test at best a model to store, analysing your performance, in front of several types of existing geographic data. The aim of this paper is to show some needs to know developed systems performance, or in developing, which purpose is digital geographic metadata management.

Keywords: Geographic Information Systems, Geoprocessing, Geography, Databases, Computation.

Bibliografia

ANDJELIC, T. e WORBOYS, M. 1996. Version management for GIS in a distributed environment in Innovations in GIS 3. Chapter Six. Editor David Parker. Selected papers from The Third National Conference ou GIS Research UK (GISRUK), Taylor and Francis.

BLOTT, S. e VCKOVSKI, A. 1995. Accessing Geographical Metafiles through a Database Storage System Lectures Notes in Computer Science 951 - Proceedings of 4th International Symposium, SSD '95 - Portland, ME, USA, August 6-9, Springer.

BLOTT, S.; RELLY, L. \& SCHEK, H. 1996. An Open Abstract-Object Storage System SIGMOD RECORD '96. Montreal.

CRISPIM, E. M. H. 1991. Avaliação de Métodos para o Desenvolvimento de Software. Dissertação de Mestrado. Rio de Janeiro, Programa de Engenharia de Sistemas e Computação - COPPE - UFRJ. 
ETTER, T. 1995. Geospatial Metadata: Standards and Storage-Mangement Considerations Diplomarbeit WS 94/95. Zürich.

FGDC. 1994a. Cadastral Standards for the National Spatial Data Infrastructure. Reston, Federal Geographic Data Committee (FGDC) - Cadastral Subcommittee - Technical Advisory Group.

1994b. Content Standards for Geospatial Metadata. Reston, Federal Geographic Data Committee (FGDC) - U.S. Geological Survey.

1994c. Guidelines for Implementing the National Geospatial Data Clearinghouse. Reston, Federal Geographic Data Committee (FGDC) - Version 1.0 - U.S. Geological Survey.

JACOBSON, B. M.; JENNINGS, G D. \& STALLINGS, C. 1994. Multi-User / Multi-Purpose GIS Databases.Computers in Agriculture.Proceedings of The 5th International Conference. Orlando, ASAE: American Society of Agricultural Engineers.

MARTIN D. 1991. Data storage. In: Geographic Information Sustems and their Socioeconomic Applications. N. York:Routledge

MSC. 1991. Towards a Coordinated Spatial Data Infrastructure for the Nation. Mapping Science Committee (MSC).

MURRAY, D. e LUTZ, D. 1994. SAIF: An Object Oriented Archival and Interchange Format for the Future. Surrey: Safe Software Inc.

RIBEIRO, G P. e MATTOSO, M. L. Q. 1995a. Bancos de Dados de Imagem: aspectos sobre armazenamento e recuperação de dados. Monografia do Curso de Pós-Graduação (Doutorado). em Engenharia de Sistemas e Computação. Rio de Janeiro: COPPE / UFRJ. 1995b. Bancos de Dados de Imagem: Metadados e Análise Temporal. Monografia do Curso de Pós-Graduação (Doutorado) em Engenharia de Sistemas e Computação COPPE / UFRJ.

1995c. Bancos de Dados de Imagens de Satélite: aspectos sobre Armazenamento, Recuperação de Dados e Linguagens de Consulta. Trabalho apresentado no XVII Congresso Brasileiro de Cartografia - Salvador (BA), p. 1031-1041 (Vol.4).

1995d. Bancos de Dados de Imagens de Satélite. Relatório Técnico ES-386/96. Programa de Engenharia de Sistemas e Computação. Rio de Janeiro: COPPE / UFRJ. 1996. Metadados geoespaciais digitais e bibliotecas cartográficas digitais. Simpósio Internacional sobre Novas Tecnologias Digitais em Geografia e Cartografia (GEODIGITAL' 96).

RIBEIRO(B), G P.; SOUZA, J. M. \& FREITAS, A. L. B. 1996. Digital Geospatial Metadata: an Brazilian Case of Federal Databases. $1^{\text {st }}$ IEEE Metadata Conference. Silver Spring.

SOUZA, J. M.; RAMIREZ, M.; FERRARI, R. \& DUARTE, M. P. C. 1993. Uma arquitetura organizacional para Sistemas de Informações Geográficas Orientados a Objetos. IV Conferência Latinoamericana sobre Sistemas de Informações Geográficas. São Paulo, II Simpósio Brasileiro de Geoprocessamento - EP/USP.

STRAUCH, J. C. M.; MATTOSO, M. L. Q. \& SOUZA, J. M. 1996. Interoperabilidade de bases de dados espaciais heterogêneas e distribuídas. I Semana Estadual de Geoprocessamento/RJ. I SEGeo-RJ. 